\title{
A game of common-pool resource management: Effects of communication, risky environment and worldviews
}

\author{
Peter Bednarik $^{* a, b}$, Joanne Linnerooth-Bayer ${ }^{\mathrm{a}}$, Piotr Magnuszewski ${ }^{\mathrm{a}, \mathrm{c}}$ and \\ Ulf Dieckmann $^{\mathrm{a}}$
}

\begin{abstract}
The 'tragedy of the commons' has been investigated for several decades. At its centre is the question whether a common resource will collapse under over-exploitation. The isolated analysis of one resource has many conceptual benefits, yet in reality resources and welfare are intertwined. In this paper, we investigate a situation where a resource which is exploited for profit has the additional feature of protecting against risk. Our main question is whether participants in an experimental game will prioritize such additional feature over maximizing profit and, if so, to what extent. Therefore, we designed a forest-harvesting game: Participants can harvest trees to generate income, and at the same time the forest serves as a protection against floods. Communication has been shown to play a vital role in managing commons. Our second aim is to test the importance of communication when the resource functions as a device of protecting against external risk. Lastly, we introduce a new perspective to the tragedy of the commons literature. Specifically, we investigate how the anthropologically motivated theory of risk perception (often called Cultural Theory) correlates with behaviour in our economic game. We believe that there is much potential in combining insights from these separate disciplines.
\end{abstract}

Keywords: tragedy of the commons, social dilemma, cooperation, behavioural experiment, cultural theory

\footnotetext{
*Corresponding author: bednarik@iiasa.ac.at

${ }^{a}$ International Institute of Applied Systems Analysis (IIASA), Schlossplatz 1, A-2361 Laxenburg, Austria

${ }^{b}$ Vienna University of Economics and Business, Welthandelsplatz 1, A-1020 Vienna, Austria

${ }^{\mathrm{C}}$ Centre for Systems Solutions, Jaracza 80b/10, 50-305 Wrocław, Poland
} 


\section{Introduction}

The sustainable use of common resources is one of the major challenges humanity has to face this century. Such resources include global climate, clean air, civil security, social security, the internet, and all living resources with shared ownership, such as stocks of fish and game. In many situations, common resources can be accessed by individuals without or with low restrictions. Therefore, they are threatened by over-exploitation. In the worst case, this may lead to the collapse of the entire resource, to a "tragedy of the commons". This term was coined by the influential work by Garret Hardin (Hardin 1968), where it is argued that such a tragedy is inevitable in many cases. Indeed a simplified game-theoretic model of such a situation, the public goods game (PGG), permits only selfish exploitation as equilibrium. Why then can we observe that many real commons can be managed consistently and without collapsing for a significant amount of time?

In the extended research on this topic, two classes of explanations arose. The first explains the gap between theory and observation by questioning the classical assumption of purely self-interested agents (homo oeconomicus). Instead of maximizing their expected utilities, individuals are suspected to have different goals. Prominent examples are risk preferences (Kahneman and Tversky 1979, Raub and Snijders 1997, Gintis 2000, Holt and Laury 2002, Hilbe et al. 2013), pro-social preferences (Fehr and Schmidt 1999, Fehr and Fischbacher 2002, Bénabou and Tirole 2006) or essentially different rationalities (Douglas and Wildavsky 1983, Thompson et al. 1990). In the second class of explanations, it is argued that realistic common goods situations often exhibit additional features or restrictions which are essential for maintaining cooperation. This means that even self-interested agents can cooperate if there are suitable mechanisms which ensure that cooperation pays off in the long run. Specifically, behaviour in a particular PGG may have impact on repeated interactions with the same social partners, or affect one's reputation (e.g., Axelrod and Hamilton 1981, Nowak and 
Sigmund 1998, Leimar and Hammerstein 2001, Panchanathan and Boyd 2004, Berger 2011). Monitoring and sanctioning systems can provide incentives to support or enforce cooperative behaviour (Fehr and Gächter 2002, Hauert et al. 2007, Maier-Rigaud et al. 2010, Rustagi et al. 2010, Sigmund et al. 2010, Zhang et al. 2014, Chen et al. 2015). In this case, models have shown that even self-interested agents should behave cooperatively under many circumstances, because it is in their own long-term interest. It is likely that both classes of explanations are relevant to understand the many examples of non-collapsing common goods situations in the real world.

Our main goal is to shed light on mechanisms that govern human-environment interactions. In the traditional tragedy of the commons, the tragedy consists of society losing one particular resource due to over-exploitation. However, in many typical environmental issues, this is not the main problem. Instead, it is the consequences on the environment: We may be less worried about the profits of the forest industry than about the extended effects of deforestation on the rest of the world. Surprisingly, literature on economic experiments is largely focused on direct effects of a single resource. We designed an experimental game in order to see how awareness of such additional effects affects sustainable management of a resource. In the long run, we aim to build upon the present work to enrich the concept of present economic experiments in a way that goes beyond overly simplistic decisions. In an attempt to ensure tractability and an incremental accumulation of insights, this work shall serve as the first step in that direction. Therefore, the multiplicity of decisions is still rather limited compared to realistic scenarios. Specifically, we added exactly one additional layer of complexity beyond the well-studied common-pool resource game: The resource gathered for profit has the additional feature of protecting against external risk so that its depletion increases that risk.

Our first and main research question is whether participants will prioritize such a second feature over maximizing profit and, if so, to what extent. To embody such a situation, we use the context of a forest-harvesting game: participants can harvest trees from a stylized forest to 
generate income. Additionally, the forest serves as a protection against floods (e.g., EEA 2015). However, we chose values such that the damage from floods is relatively low. Thus, the gametheoretic structure of a social dilemma is not resolved, in particular, the addition of flood risk does not change the Nash equilibrium of the game (see Appendix A3). According to Kahnemann and Tversky's (1979) prospect theory, some individuals are likely to weight losses stronger than gains (loss aversion). We therefore expect that the risk of floods will make participants more careful and hence more efficient in managing the resource.

Communication has been identified as a major driving force to resolving the tragedy of the commons (Cardenas et al. 2004). Thus, our second hypothesis concerns the role of communication in our setting. Apart from hoping to provide a replication of the cooperationenhancing effect of communication, we want to identify whether the effect of our first hypothesis holds with and/or without the possibility of communication.

In addition, we introduce a different angle to the subject using the theory of plural rationality (also called cultural theory; see, e.g., Thompson et al. 1990, Linnerooth-Bayer et al. 2003, Verweij and Thompson 2006) ${ }^{4}$, which postulates that stakeholder discourses (or voices) are plural but limited in number. The discourses stem from different social contexts, which, in turn, are shaped by the ways in which people organize, perceive and justify their social relations. The theory argues that there are four ways of organizing (thus the limited number of discourses): hierarchy, individualism, egalitarianism and fatalism. Individual tendencies to accord with these four ways we shall call worldviews. It is very likely that such worldviews affect behaviour in social dilemma games. However, due to lack of overlap between the respective disciplines, little is known about such a possible connection. As a first step into filling this gap, we used a questionnaire on the worldviews of participants and correlated it with

\footnotetext{
${ }^{4}$ Originally developed by Mary Douglas (1978) as a "heuristic device" or "analytical scheme", it is a cultural theory - a theory of cultural bias, to be precise - but it all too easily gives the mistaken impression that it is culture that is doing the explaining. "Plural rationality" avoids that; it also helps position this theory in relation to those - rational choice and post-structuralism - that it challenges.
} 
98 their behaviour in the game. Since this is - to our best knowledge - the first study connecting these scientific areas, it is difficult to give specific hypothesis on the direction of correlations. Further work must address this question more specifically in order to disentangle correlation and causation of worldviews and pro-social behaviour. 


\section{Material and methods}

\subsection{The participants}

We conducted computerized experiments with 320 students, tested in fall 2015 and spring 2016. Students were recruited via the online recruitment system ORSEE (Greiner 2004) and represented a broad range of disciplines. They were composed of $48.7 \%$ females. Upon arrival participants were randomly seated in front of computers separated by opaque partitions. Participants were informed via written instructions (see Appendix A1) about the game rules and were informed that their decisions would be made anonymous to the other participants and the experimenters. They were forbidden to communicate except via computers. To allow for in-game identification while ensuring anonymity with regard to their real identity, participants were given pseudonyms (in each of the groups of five we used the names Helike, Ferin, Ananke, Metis and Kalisto, which are names of moons of the solar system, cf. Bednarik et al. 2014). Sessions lasted approximately 70 minutes and participants earned $€ 17.12 \pm € 4.96$. After the game, participants filled out a questionnaire indicating their worldviews on various topics (see Appendix A4. For the questionnaire analysis, only part A of the questionnaire was used.).

\subsection{Game rules and treatments}

In fall 2015, we conducted eight groups for each of the five treatments (described below) with five participants in each group. In spring 2016, we conducted eight groups for each of three treatments, one of which was the same as in 2015. Thus, we have in total seven treatments, each with eight groups except one treatment with 16 groups. The reason is that the initial five treatments all included the option to communicate via chat-box, which we controlled for in the second cohort of experiments. We shall first explain the detailed rules of the simplest treatment and later the additional rules of the subsequent treatments: 
Time was allocated for the participants to read the written instructions and to ask

questions, after which the experimenter started the game. Each round of the game consisted of two phases: i) the operations phase and ii) the results phase. The participants were informed about the total length of the game, which was 20 rounds. In the operations phase, participants could harvest trees from a forest that was represented by an 8x10 matrix (Figure 1), where each cell or patch contained one tree. Initially, all patches were filled by trees. For each harvested tree, the participants received $€ 0.10$. Apart from the show-up fee $(€ 5)$, this was the only source of the participants' income. In the operations phase, participants could continuously harvest trees for one minute. In the results phase, which also lasted one minute, participants had the opportunity to check their own and other players' earnings, and prepare their strategy for the next round. At the beginning of each new round, a specified proportion of the forest regrew. For each existing tree, one additional tree regrew, up to a maximum of 80 trees. Additionally, 5 trees grew each round independently of existing trees unless the maximum of 80 trees was reached already.

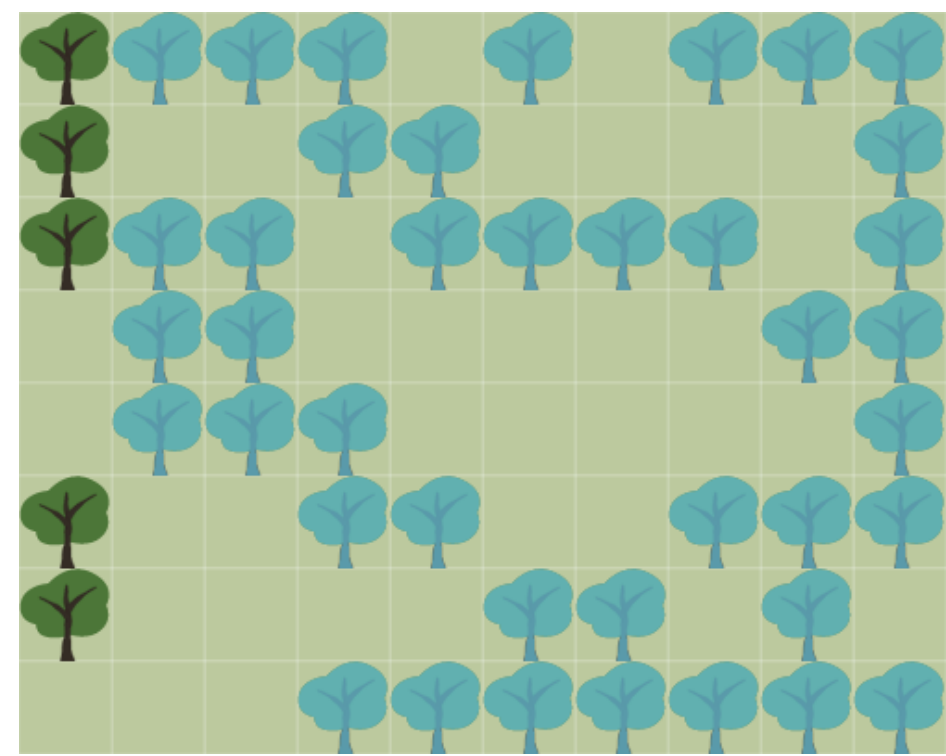

Figure 1. The forest game. In each group, five players may click on a tree to harvest it and receive profit points. In treatments with rainfall, trees serve an additional function: to protect from floods. If the rainfall intensity exceeds the size of the forest, players will be subject to flood damage, reducing their payoffs. In the example shown above, the rainfall is fully absorbed by the sufficiently large forest and no damage occurs. 
In five treatments, participants were permitted to communicate throughout the entire

experiment via chat-box, using their pseudonyms. During each round, a certain (fixed or random as explained below) amount of precipitation occurred. The uncut forest served as flood protection. If the precipitation exceeded the capacity of the uncut trees to absorb the water, a flood occurred and reduced participants' payoffs. The treatment without the possibility of floods was labelled NOFLO. The other treatments with chat-box followed a $2 \times 2$ design. Rainfall could happen REGularly or IRRegularly and the resulting flood losses could be distributed EQually or Unequally (hence these treatments were labelled REGEQ, REGUN, IRREQ and IRRUN). In all treatments except NOFLO, rainfall occurred at the end of each operations phase. The rainfall intensity was either 25 for REG treatments or a random number between 0-50 for IRR treatments (the sequence of the numbers was pre-generated with the condition that the mean was exactly 25). The uncut forest had the capability of absorbing runoff from the rain: Each tree reduced the potential flooding intensity by one point. If the resulting intensity was reduced to 0 or below, no flood occurred. Otherwise, the resulting flood inflicted damage which was deducted from players' payoffs. The total flood damage was estimated as the difference between the rainfall intensity (0-50) minus the number of uncut trees. The flood damage was distributed to the players (reducing their payoffs) either equally (in EQ treatments) or unequally (in UN treatments). In the latter case, one player bore $50 \%$ of the flood damage, one player $25 \%$, one player $15 \%$, one player $10 \%$ and one player $0 \%$. This damage distribution was different in each round (randomly, but modified to ensure the same sums of weights over all rounds for all players). Regardless of whether the distribution of damage was equal or unequal the flood damage did not outweigh individual profits from harvesting trees. Even if the forest was at its minimal water carrying capacity, each harvested tree gave one point to the harvester while it caused only between 0 and 0.5 points (on average: 0.2 points) damage.

In addition to these five treatments, we ran the treatments NOFLO and REGEQ without the chat-box, which we shall refer to as NOFLO-NC and REGEQ-NC. In the second cohort we 
also ran another 8 groups with the REGEQ treatment. In summary, we had 7 treatments, with 8 groups of 5 players in each treatment, except for REGEQ, where we had 16 groups. Thus, we had $(7+1) \times 8=64$ groups, meaning that a total of 320 participants were tested.

\subsection{Statistical analysis}

Except where specifically indicated, we used group averages as our statistical units since the behaviour of individual participants within each group was interdependent. For statistical analysis R 3.2.1 (R Core Team 2013) was used. Probabilities are reported as two tailed at a 5\%significance level. 


\section{Results}

\subsection{Flood risk reduces over-harvesting}

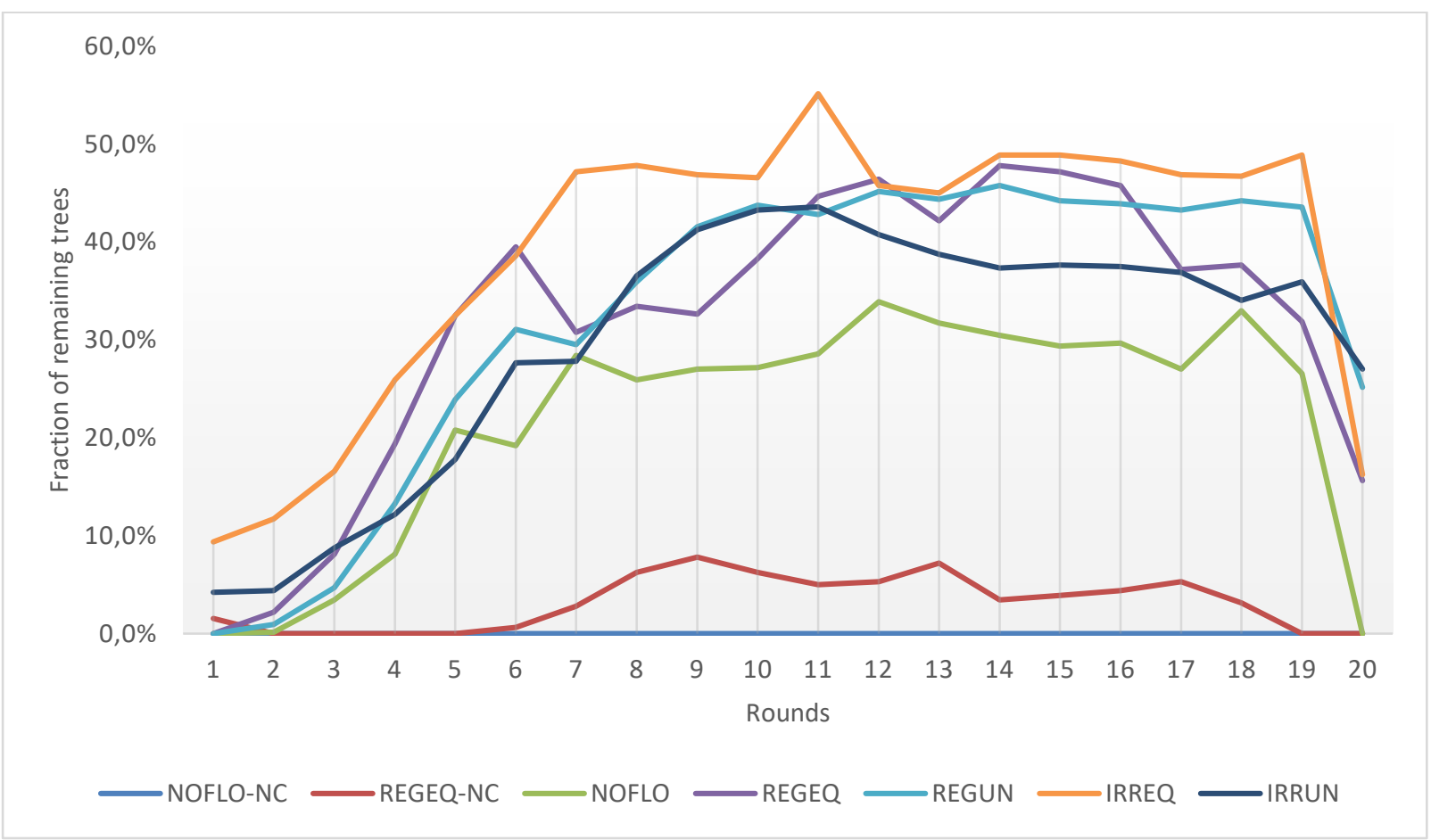

Figure 2. Average fraction of remaining trees per round for each of the seven treatments (abbreviations see in methods section 2.2). Clearly, treatments without the possibility of chat communication perform poorly. Further, the groups' ability to maintain a larger number of trees is also affected by the possibility of floods.

Average forest condition ( $\%$ of uncut trees) is higher in the flood treatments than in the no flood treatment $(27.47 \pm 15.12$ vs. $10.77 \pm 14.23$, Wilcoxon rank-sum test: $W=171, p<0.001$, see Fig. 2), which suggests that the risk of floods mitigates the social dilemma of forest management. However, the resulting increase in efficiency was not sufficient to compensate for occasional losses from floods. Hence, the final payoffs were not significantly different in treatments with and without floods. Interestingly, however, individual payoffs were significantly negatively correlated with individual harvest rate (the percentage each individual harvested from each round's initial forest condition, averaged over all 20 rounds, Pearson's $r=0.179, \mathrm{df}=318, \mathrm{p}=$ 0.0013). This can be interpreted as evidence for "nice guys finish first", because high individual harvest rates often imply an early deforestation. After that, a collective agreement to abstain 
$226=38, \mathrm{p}=0.108)$.

from harvesting in order to regrow the forest is difficult. As a result, individual earnings of all group members can be reduced.

\subsection{Uncertainty impacts behaviour}

Contrary to the differences to the NOFLO-treatment, all treatments with floods showed similar behaviour when averaged over the entire game duration. However, on a closer look there exist clear differences in behaviour of groups in treatments with irregular rainfall compared to the rest. In the first round, nearly all (57 of 64) groups deforested the entire area. Of the seven groups that did not deforest the entire area, five belonged to the irregular treatments (IRREQ or IRRUN). This is especially remarkable because in the first round, players had little time to gather experience about the game or to communicate. Evidence for risk-averse behaviour is found when looking at the optimal harvesting rate. The maximum regeneration in all treatments was 42 trees per round which could be achieved by leaving 38 trees and harvesting the rest. Groups in the irregular treatment tended to leave more trees than 38 significantly more often than other groups $(36.56 \pm 26.57$ vs. $15.83 \pm 15.58$, Wilcoxon rank-sum test: $W=281.5, p=$ 0.0132). Because rainfall was unpredictable in these treatments (IRREQ and IRRUN), it is likely that these groups tried to build up a buffer to prevent flood damage.

\subsection{No cooperation without communication}

As expected, communication appears to play a vital role in managing common goods: Average forest condition is higher in the chat treatments than in the no chat treatments $(30.53 \pm 12.01 \mathrm{vs}$. 1.57 \pm 4.38 , Wilcoxon rank-sum test: $\mathrm{W}=29, \mathrm{p}<0.000001$, cf. Fig. 2). Further, among those treatments with chat, we find a tendency that longer chat communication correlates with higher levels of cooperation, i.e., lower levels of over-harvesting (Pearson's $r=0.258, t=1.6456$, df 
As mentioned in the section 1, we made a first step for building a bridge to the

sociologically/anthropologically driven theory of plural rationalities, which states that social interactions depend on four distinct worldviews: hierarchy, individualism, egalitarianism and fatalism. We applied a post-game questionnaire to elicit worldviews of the players, and compared the four worldviews with the group outcomes. The questionnaire allowed us to score participants on each of the worldviews on a scale 1-10. Linking group averages of the individual scores with average group forest condition resulted in the correlations show in Figure 3. Egalitarian affinities improve a group's ability to preserve the forest (positive correlation), whereas individualistic and fatalistic affinities have a slightly opposite effect (negative correlations). This finding is in line with the theory's predictions, because preserving environment and sharing resources equally are assumed to be predominantly egalitarian traits.

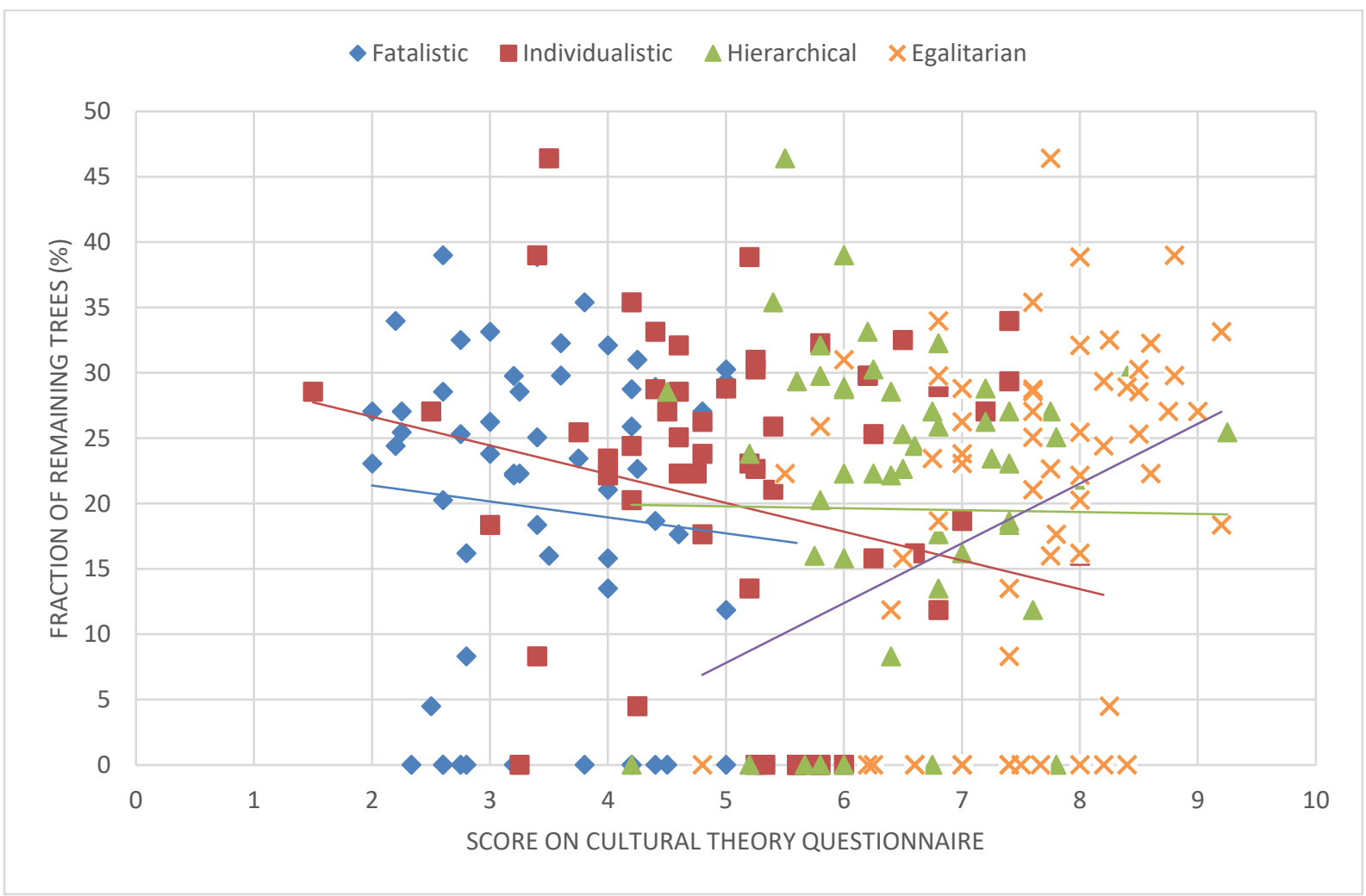

Figure 3. Group averages of worldview scores plotted against group averages of remaining trees. Each circle represents a group's average score on one of the four worldviews plotted against the same group's average number of remaining trees. 


\section{Discussion}

The conducted experiments show the influence of shared benefits, communication, and individual worldviews on the outcome of a common-pool resource management game. The addition of group-level benefits for reducing the harvest rate improves the group outcomes in terms of the forest sustainability in all treatments with and without communication. One explanation for this result is based on risk aversion (Kahneman and Tversky 1979). The flood damage affects everyone (although not always to the same extent, depending on the treatment) which means that individual over-harvesting does not only destroy the potential long-term benefits, but also leads to direct costs in terms of flood damage. Although the addition of flood risk does not change the dominant individual strategy leading to overharvesting, by reframing the context, it may trigger different heuristics for making harvesting decisions (Shaffer et al. 2011). The potential of flood losses, by adding an external source of uncertainty (besides uncertainty related to decisions of other players), increases cognitive processing required to assess the decision with a best individual outcome. In such situations, players may rely more on simple heuristics (Todd and Gigerenzer 2000) - in this case lowering the harvest. It has been demonstrated that such simple heuristics can perform better than decisions guided by optimal outcomes, in highly uncertain situations (Pflug et al. 2012). In future studies we will try to assess to what extent participants are aware of the game-theoretic individual optimum decisions and if additional risk induces a deviation from this decision or, alternatively, if they switch to a different decision mode using one of simple heuristics.

The results linking individual cultural theory-based characteristics with the behaviour in the common-pool resource experiments show that egalitarian worldviews are correlated with more sustainable strategies. This demonstrates a potential for cultural theory to add another classification to the repertoire of other-regarding preferences (e.g., Fehr \& Schmidt, 1999) that may explain and enrich our understanding of how different combinations of player types may lead to more sustainable behaviour. A number of studies investigating cultural effects on the 
behaviour of participants in experimental economics games have revealed interesting patterns (Prediger et al. 2011, Ghate et al. 2013). Cultural theory seeks to explain cultural differences by addressing four "ways of life" that are present within any specific culture (as addressed by anthropological and psychological literature). The results we obtained are consistent with the understanding of the key instruments used by different solidarities: individualists relying on incentives, hierarchists relying on rules and egalitarians relying on norms. In the absence of incentives and rules in the game design, egalitarians are more likely to overcome the social dilemma and achieve more sustainable outcomes. At the same time, a well-functioning group which results in good forest management could make participants respond stronger to egalitarian values. Further research is needed to investigate the causal interactions between participants' behaviour and worldviews.

Finally, it is important to emphasize the dynamic form of resource representation in the experiments. Although it is now common to investigate the common-pool resource situation with the static resource model (as in public goods games), such a representation misses the critical component of the resource dynamics. The participants' harvesting decisions affect the resource state that may lead to a case-specific trajectory, adding the history dimension that may in turn induce path-dependency. Effectively, participants' decisions depend not only on decisions of others from previous rounds but also on the state of the resource itself. This additional complexity can increase the difficulty of identifying the players' strategies; however, the findings better represent actual decision-making in common-pool resource situations.

\section{Acknowledgements}

The experiment was conducted at the Vienna Centre for Experimental Economics (VCEE), University of Vienna. We thank the students for participating in the experiments. Discussions with Karl Sigmund and Michael Thompson are gratefully acknowledged. 


\section{References}

Axelrod, R., and W. D. Hamilton. 1981. The evolution of cooperation. Science 211:1390-1396.

Bednarik, P., K. Fehl, and D. Semmann. 2014. Costs for switching partners reduce network dynamics but not cooperative behaviour. Proceedings of the Royal Society of London B: Biological Sciences 281:20141661.

Bénabou, R., and J. Tirole. 2006. Incentives and prosocial behavior. The American economic review 96:1652-1678.

Berger, U. 2011. Learning to cooperate via indirect reciprocity. Games and Economic Behavior 72:30-37.

Cardenas, J.-C., T. Ahn, and E. Ostrom. 2004. Communication and co-operation in a commonpool resource dilemma: a field experiment. Pages 258-286 Advances in Understanding Strategic Behaviour. Springer.

Chen, X., T. Sasaki, Å. Brännström, and U. Dieckmann. 2015. First carrot, then stick: how the adaptive hybridization of incentives promotes cooperation. Journal of The Royal Society Interface 12:20140935.

Douglas, M., and A. Wildavsky. 1983. Risk and culture: An essay on the selection of technological and environmental dangers. Univ of California Press.

Fehr, E., and U. Fischbacher. 2002. Why social preferences matter-the impact of non-selfish motives on competition, cooperation and incentives. The economic journal 112:C1C33.

Fehr, Ernst, and Simon Gächter. 2002 "Altruistic punishment in humans." Nature 415.6868: 137.

Fehr, E., and K. M. Schmidt. 1999. A theory of fairness, competition, and cooperation. Quarterly journal of Economics:817-868. 
Ghate, R., S. Ghate, and E. Ostrom. 2013. Cultural norms, cooperation, and communication: Taking experiments to the field in indigenous communities. International Journal of the Commons 7.

Gintis, H. 2000. Beyond Homo economicus: evidence from experimental economics. Ecological economics 35:311-322.

Greiner, B. 2004. The online recruitment system ORSEE 2.0 - A guide for the organization of experiments in economics.Working Paper Series in Economics, 10. University of Cologne: Cologne, Germany.

Hardin, G. 1968. The tragedy of the commons. Science 162:1243-1248.

Hauert, C., A. Traulsen, H. Brandt, M. A. Nowak, and K. Sigmund. 2007. Via freedom to coercion: the emergence of costly punishment. Science 316:1905-1907.

Hilbe, C., M. A. Chakra, P. M. Altrock, and A. Traulsen. 2013. The evolution of strategic timing in collective-risk dilemmas. PLoS ONE 8:e66490.

Holt, C. A., and S. K. Laury. 2002. Risk aversion and incentive effects. American economic review 92:1644-1655.

Kahneman, D., and A. Tversky. 1979. Prospect theory: An analysis of decision under risk. Econometrica: Journal of the Econometric Society:263-291.

Leimar, O., and P. Hammerstein. 2001. Evolution of cooperation through indirect reciprocity. Proceedings of the Royal Society of London B: Biological Sciences 268:745-753.

Linnerooth-Bayer, J., M. Mace, R. Verheyen, and K. Compton. 2003. Insurance-related actions and risk assessment in the context of the UNFCCC.in background paper prepared for the UNFCCC Secretariat (UNFCCC, Bonn, 2003). http://unfccc. int/meetings/workshops/other_meetings/items/1043. php.

Maier-Rigaud, F. P., P. Martinsson, and G. Staffiero. 2010. Ostracism and the provision of a public good: experimental evidence. Journal of Economic Behavior \& Organization 73:387-395. 
Nowak, M. A., and K. Sigmund. 1998. Evolution of indirect reciprocity by image scoring. Nature 393:573-577.

Panchanathan, K., and R. Boyd. 2004. Indirect reciprocity can stabilize cooperation without the second-order free rider problem. Nature 432:499-502.

Pflug, G. C., A. Pichler, and D. Wozabal. 2012. The 1/N investment strategy is optimal under high model ambiguity. Journal of Banking \& Finance 36:410-417.

Prediger, S., B. Vollan, and M. Frölich. 2011. The impact of culture and ecology on cooperation in a common-pool resource experiment. Ecological economics 70:1599-1608.

R Core Team. 2013. R: A language and environment for statistical computing. R Foundation for Statistical Computing, Vienna, Austria.

Raub, W., and C. Snijders. 1997. Gains, losses, and cooperation in social dilemmas and collective action: The effects of risk preferences. Journal of Mathematical Sociology 22:263-302.

Rustagi, D., S. Engel, and M. Kosfeld. 2010. Conditional cooperation and costly monitoring explain success in forest commons management. Science 330:961-965.

Shaffer, D. M., S. M. Krauchunas, M. Eddy, and M. McBeath. 2011. Heuristics: The Foundations of Adaptive Behavior.in Oxford University Press.

Sigmund, K., H. De Silva, A. Traulsen, and C. Hauert. 2010. Social learning promotes institutions for governing the commons. Nature 466:861-863.

Thompson, M., R. Ellis, and A. Wildavsky. 1990. Cultural theory. Westview Press.

Todd, P. M., and G. Gigerenzer. 2000. Précis of simple heuristics that make us smart. Behavioral and brain sciences 23:727-741.

Verweij, M., and M. Thompson. 2006. Clumsy solutions for a complex world: Governance, politics and plural perceptions. Springer. 
Zhang, B., C. Li, H. De Silva, P. Bednarik, and K. Sigmund. 2014. The evolution of sanctioning institutions: an experimental approach to the social contract. Experimental Economics 17:285-303. 